\title{
Métodos de evaluación de la Dimensión Vertical Oclusal.
}

\section{Methods of evaluation of the Occlusal Vertical Dimension.}

\author{
Juan Carlos Espinosa-Valarezo ${ }^{1 *}$, Roberto Irribarra-Mengarelli², Héctor González-Bustamante ${ }^{2}$
}

1. Cirujano Dentista U. De Cuenca, Ecuador, Alumno Programa Especialización Universidad Andrés Bello.

2. Cirujano Dentista U. De Chile, Chile, Universidad Andrés Bello, Sociedad de Prótesis Y Rehabilitación Oral de Chile.

${ }^{*}$ Correspondencia autor: Juan Carlos Espinosa | Dirección: San Eugenio 990 dep 1305, Ñuñoa, Santiago Chile. | Teléfono +56 969060183 | Email: juanesvala@hotmail.com

Trabajo recibido el 26/06/2017. Aprobado para su publicación el 10/09/2017

\begin{abstract}
RESUMEN
Antecedentes: La evaluación de la Dimensión Vertical Oclusal, es una fase importante en el diagnóstico y planificación del tratamiento que determina las relaciones estéticas y funcionales entre los maxilares. Objetivo: Conocer los métodos que ayuden a determinarla, ventajas y desventajas de cada uno, y los parámetros para reestablecerla. Materiales y Métodos: Se recopiló información científica desde las bases electrónicas Medline/Pubmed, EBSCO y Schoolar Google desde Enero del 2010, hasta Marzo del 2016 tanto en Inglés y Español, utilizando las palabras Dimensión Vertical Oclusal, Altura Facial Inferior y Espacio de Inoclusión Fisiológica, se leyeron los títulos y resúmenes para descartar los artículos no relacionados, eliminando los repetidos. Resultados y Discusión: La relación proporcional entre los segmentos de la cara varían de acuerdo a los estudios, de estos, las mediciones antropométricas presentan el mayor número de discrepancias, y sus resultados no pueden ser aplicados a todos los individuos.

Conclusiones: no hay un método único que determine con exactitud la Dimensión Vertical Oclusal, ni evidencia científica que respalde la asociación de Trastornos Témporo Mandibulares con variaciones en la misma, incrementos de $5 \mathrm{~mm}$ son predecibles y aunque pueden manifestar incomodidades iniciales a los pacientes, son auto limitante y remiten en pocos días.
\end{abstract}

\section{PALABRAS CLAVE}

Dimensión vertical oclusal; Altura facial inferior; Espacio de inoclusión fisiológica

Rev. Clin. Periodoncia Implantol. Rehabil. Oral Vol. 11(2); 116-120, 2018.

\section{ABSTRACT}

Background: The evaluation of the Occlusal Vertical Dimension is an important phase in the diagnosis and treatment planning that determines the aesthetic and functional relationships between the jaws. Objective: To know the methods that help to determine it, the advantages and disadvantages of each one, and the parameters to reestablish it. Materials and Methods: Scientific information was collected from the electronicdatabases Medline / Pubmed, EBSCO and Schoolar Google from January 2010 toMarch 2016 in both English and Spanish, using the words Occlusal Vertical Density,Lower Facial Height and Physiological Inoculation Space, Titles and abstracts wereread to discard unrelated articles, eliminating repeated ones. Results and Discussion:The proportional relationship between the segments of the face varies according tothe studies; out of these, the anthropometric measurements present the greatest numberof discrepancies, and their results can not be applied to all individuals. Conclusions: There is no single method that accurately determines the occlusal vertical dimension,nor scientific evidence supporting the association of temporomandibular disorders with variations in it; increments of $5 \mathrm{~mm}$ are predictable and although they may cause initial discomforts to patients, they are self-limiting and diminish in a few days.

KEYWORDS

Occlusal vertical dimension; Inferior facial height; Physiological space inocclusion

Rev. Clin. Periodoncia Implantol. Rehabil. Oral Vol. 11(2); 116-120, 2018.

\section{INTRODUCCIÓN}

El glosario de términos odontológicos define a la Dimensión Vertical (DV) como la distancia entre dos puntos anatómicos seleccionados ${ }^{(1)}$, uno sobre un elemento fijo en el maxilar y otro sobre un elemento móvil mandibular (usualmente uno ubicado en la punta de la nariz y otro sobre la barbilla), por lo tanto, la Dimensión Vertical Oclusal (DVO) corresponderá a la distancia medida entre dichos puntos cuando las piezas dentales están en contacto oclusal ${ }^{(1,2)}$.

Durante el proceso de valoración de un paciente tanto en la práctica odontológica en general como en la especialidad de Rehabilitación
Oral en particular, la DVO es uno de los primeros parámetros en ser establecidos, su determinación es una fase importante en la etapa de diagnóstico y en la posterior planificación del tratamiento(3) procurando establecer relaciones estéticas y funcionales de los maxilares, tomando en consideración que cualquier alteración de esta medida durante los procesos restaurativos es insegura para el sistema estomatognático ${ }^{(4)}$, si bien, la pérdida de la DVO es una posible consecuencia del desgaste dentario, esta también puede estar mantenida por un mecanismo de compensación dentoalveolar que envuelve la sobreerupción del diente desgastado ${ }^{(1,2,5)}$, expansión del hueso alveolar y la acción muscular ${ }^{(4)}$, por ello la importancia de registrarla previamente y en base a su evaluación 
proceder a mantenerla, recuperarla o aumentarla de acuerdo a las necesidades de cada caso. La disminución de la DVO puede desarrollar lesiones tales como Queilitis Angular, desarmonía de la estética facial de tercio inferior de la cara, signos de vejez prematura ${ }^{(2)}$, pseudprognatismo mandibular $^{(1)}$ y para algunos autores incluso desordenes temporomandibulares $^{(5)}$, las consecuencias reportadas por incrementar la misma corresponden a hiperactividad de los músculos masticatorios, aumento de la fuerza oclusal, bruxismo ${ }^{(1,4)}$, dificultad en la fonación y deglución, sensibilidad dental debido a las fuerzas traumáticas, reabsorción ósea patológica, alargamiento del rostro, así como, expresión facial de cansancio ${ }^{(3)}$. Existen numerosos procedimientos para la determinación de la $\mathrm{DV}^{(2)}$, algunos autores establecen que esta debe ser restaurada probablemente al mismo valor que tenía antes de producirse la disminución de la mis$\mathrm{ma}^{(3,6)}$, y por ello se han utilizado algunos métodos para determinar las relaciones verticales de la mandíbula con respecto al maxilar tales como: la posición de reposo fisiológico mandibular, deglución, fonéticos, y estéticos, así como en los que se incluyen medidas faciales, registros pre extracción, cefalometrías ${ }^{(7)}$ o métodos electromiográficos; encontrar un procedimiento fiable para determinar la dimensión vertical de la oclusión correcta siempre ha sido un reto para los clínicos, y desafortunadamente, no hay un procedimiento en particular universalmente aceptado que determine la dimensión vertical por si solo ${ }^{(2,6,7)}$. De acuerdo a Matsumoto ${ }^{(3)}$ la DVO puede ser dividida en Clase I: cuando está mantenida por el contacto dentario; esta situación se extiende desde la presencia del arco dental completo hasta la más extrema situación donde solo dos dientes antagonistas están en contacto, Clase II: cuando a pesar de la presencia de los dientes, ninguno de ellos toma contacto con el antagonista, por lo tanto la DVO no se puede mantener porque no hay contacto en el arco, Clase III: el contacto oclusal es totalmente ausente así como uno de los arcos es completamente edéntulo ${ }^{(3)}$, por lo tanto, los factores que se deberían considerar determinantes para incrementar la DVO constituyen principalmente la estructura dental remanente, el espacio disponible para la rehabilitación, la oclusión y la estética; si bien, los incrementos de hasta $5 \mathrm{~mm}$ son predecibles y seguros en el tiempo ${ }^{(1,8)}$, la decisión de cambiar la DVO implica necesariamente un proceso de evaluación y seguimiento al paciente hasta que en él desaparezcan los signos y síntomas de disconfort $^{(2)}$ y se haya demostrado la adaptabilidad al cambio propuesto ${ }^{(8)}$.

En base a lo planteado, el objetivo de esta revisión es describir los métodos de registro más comúnmente utilizados en la valoración de la DVO y de acuerdo a la evidencia científica determinar, si es posible, cuales son los más adecuados para determinarla en los pacientes.

\section{METODOLOGÍA}

Se realizó la búsqueda electrónica de documentos mediante el uso de buscadores científicos Medline/Pubmed, EBSCO Y Schoolar Google, utilizando una combinación de palabras clave: Dimensión Vertical Oclusal, Altura Facial Inferior y Espacio de Inoclusión Fisiológica, los filtros de búsqueda empleados constituyen artículos en inglés y español, y publicados desde Enero del 2010 hasta Marzo del 2016, de acuerdo a las palabras clave la búsqueda inicial entre todos los archivos se registraron 1189 artículos, de los cuales se seleccionaron 49 que incluyeron las palabras clave en el título, se excluyeron los que estaban repetidos ${ }^{(12)}$, quedando 37 artículos para su análisis, se procedió a la lectura de los resúmenes para clasificar a aquellos cuyo contenido guarde relación con los objetivos planteados y se seleccionaron 19 artículos, luego de revisar las citas bibliográficas se incorporó tres más que inicialmente no fueron incluidos por su fecha de publicación (1980, 2006 y 2009), cuyo contenido sirve de aporte sostenido para esta revisión. En base al objetivo planteado de determinar si hay relación entre las diversas medidas antropométricas del rostro se agruparon 5 estudios en base a criterios de inclusión específicos en los cuales dichos artículos deben incluir una población de estudio de pacientes completamente dentados a excepción de los terceros molares, con relación mandibular clase I, con rostros armoniosos y simétricos; estudios en los que describen con claridad la metodología empleada para establecer los puntos de referencia anatómicos; estudios en los que se muestre valores promedio y desviación estándar de cada uno de los registros obtenidos, se escogió los documentos en los cuales este presente la medición desde el punto Subnasal a mentón como referencia de comparación para determinar la DVO.

\section{RESULTADOS Y DISCUSIÓN}

¿Cuál es la Dimensión Vertical Oclusal (DVO) normal de una persona?

La definición explícita de DV corresponde a la medida ente dos puntos anatómicos seleccionados ${ }^{(1,5)}$, uno en el maxilar y otro en la mandíbula(9), y la DVO correspondería a la misma medición pero con relación inte- cuspal de los dientes antagonistas ${ }^{(2,3,5)}$, sin embargo, esta medición debe guardar armonía o cierta relación con otras estructuras de la cara para que sea considerada armónica, estos conceptos de armonía se remontan al siglo XV en donde artistas como Leonardo Da Vinci promovían dicha proporción estética(6); en base a estos conceptos algunos investigadores realizaron sus primeros estudios de confección de prótesis dentales en base a la división de cuatro segmentos proporcionales de la cara ${ }^{(6)}$, estableciendo la relación del tercio inferior de la cara con una medida específica del tercio medio. En 1980 Legan HL, Burstone CJ. introducen un estudio cefalométrico en donde analizan los tejidos blandos de la cara y determinan una relación armónica entre dichos tercios con el objeto de definir parámetros estéticos aplicables en cirugía ortognática, para ello, analizaron pacientes caucásicos que no manifiesten desarmonías faciales, no hayan tenido tratamientos de ortodoncia previos y tampoco pérdida de piezas dentales, estos pacientes eran considerados armónicos a la inspección clínica, dentro de las múltiples medidas determinadas las relacionadas establecidas entre la distancia Glabela a Sub Nasal y Sub Nasal a Menton Blando guardan una proporción existente de 1:1 en

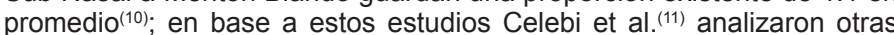
poblaciones étnicas y replicaron el estudio cefalométrico en la población turca encontrando una relación equivalente de 1,05:1 con una Desviación Estándar (DE) de 0,1; resultados similares fueron observados por Uysal et al. en una población de la misma etnia, así mismo, estudios en otras poblaciones como lo efectuados por Issacson en China, Alcalde en Japón, Jain en India del Norte, Albarakati en Arabia Saudita y Gunaid en la población Yemen ${ }^{(11)}$, establecen relaciones similares y que pueden ser observados en la tabla 1 , si bien no corresponden exactamente a la determinada en los pacientes caucásicos las discrepancias serían mínimas pero no se ha podido determinar si la variación es estadísticamente significativa(11) $^{(11}$

Tabla 1. Valores cefalométricos del tejido blando y Desviación Estándar de la altura vertical en diferentes grupos étnicos utilizando el análisis de Legan y Burstone

\begin{tabular}{|c|cccccc|}
\hline Autor & Issacson & Alcalde & Jain & Albarakati & Gunard \\
\hline Año & 1989 & 2000 & 2011 & 2011 & 2000 \\
\hline Etnia & China & Japonés & $\begin{array}{c}\text { Norte } \\
\text { de India }\end{array}$ & Saudí & Yemen \\
\hline $\begin{array}{c}\text { Altura Vertical } \\
\text { G-Sn Sn- Me }\end{array}$ & $\begin{array}{c}1.01 \\
+/-0.1\end{array}$ & $\begin{array}{c}0.9 \\
+/-0.1\end{array}$ & $\begin{array}{c}1.03 \\
+/-0.1\end{array}$ & $\begin{array}{c}1.0 \\
+/-0.09\end{array}$ & $\begin{array}{c}1.01 \\
+/-0.1\end{array}$ \\
\hline
\end{tabular}

Al realizar mediciones clínicas Helal et al. ${ }^{(12)}$ utilizando un calibrador lineal encontraron entre otras medidas, una relación parecida entre los puntos antes indicados, donde el segmento superior es $0.07 \mathrm{~mm}$ mayor al inferior, lo que sería equivalente a una relación de 1:1.01, en un estudio similar Nagpal et al. ${ }^{(6)}$ por lo contrario, encuentra una discrepancia en los puntos mencionados donde el segmento superior es $3.2 \mathrm{~mm}$ mayor que el inferior, sin embargo la edad de los pacientes estudiados es diferente; estudios antropométricos similares efectuados por Babu et al. ${ }^{(13)}$, Majeed et al. ${ }^{(14)}$ y Nasser et al. ${ }^{(15)}$ tratan de relacionar el tercio inferior del rostro con otras medidas para así establecer nuevas guías que encaminen al clínico a buscar una DVO óptima, un resumen de los resultados se muestran en la Tabla 2.

La relación proporcional entre los segmentos medio e inferior de la cara no son estándar y varían de acuerdo a los diferentes estudios ya sean a través de medidas clínicas ${ }^{(6,12,13,14,15)}$ o estudios cefalométricos ${ }^{(12)}$, la edad de los pacientes, el sexo, la etnia, e incluso el número de la población estudiada influyen para que los datos obtenidos no sean estandarizados a cada individuo, sin embargo se puede inferir de acuerdo a los hallazgos encontrados en la literatura que la relación obtenida entre los puntos Subnasal y Glabela(11), así como, la distancia entre los puntos medidos desde el canto externo del ojo hasta la comisura de la boca (Método de Willis) $)^{(12)}$ en el tercio medio de la cara, pueden ser utilizados como puntos de referencia para determinar la DVO, puesto que guardan una estrecha relación con el punto Subnasal mentón blando del tercio inferior del rostro ${ }^{(11)}$. Un resumen esquemático de la relación más proximal entre los diferentes estudios es representado en la Figura 1.

\section{Técnicas para evaluar la Dimensión Vertical Oclusal (DVO)}

En la literatura se describen variadas formas o métodos por los cuales se puede establecer la $\mathrm{DVO}^{(16,17)}$ el procedimiento clínico clásico ${ }^{(9)}$ parte con la determinación de la Dimensión Vertical Postural (DVP) el cual se corrobora utilizando los test de fonación ${ }^{(17,19)}$, de deglución ${ }^{(9)}$, o la armonía facial. Este procedimiento ha servido de punto de comparación y verificación con otros estudios ${ }^{(9,17,19)}$, sin embargo también se han mencionado los registros pre extracción ${ }^{(20)}$, magnitud del espacio de inoclusión fisiológica ${ }^{(16,17,18)}$, estudios cefalométricos ${ }^{(11,19)}$, un resumen de las principales 
Tabla 2. Promedio de medidas obtenidas así como desviación estándar de los parámetros antropométricos establecidos en pacientes dentados.

\begin{tabular}{|c|c|c|c|c|c|c|}
\hline Autor & $\begin{array}{l}\text { Número de } \\
\text { Medidas } \\
\text { Faciales }\end{array}$ & Edades & Tipos de Medidas Faciales en MIC & $\begin{array}{c}\text { Muestra en } \\
\text { pacientes dentados }\end{array}$ & $\begin{array}{l}\text { Promedio } \\
\text { en } \mathrm{mm}\end{array}$ & D.E. \\
\hline \multirow{8}{*}{ Nagpal A. 2014} & \multirow{8}{*}{8} & \multirow{8}{*}{50 a 60 años } & Glabela - Subnasal & \multirow{8}{*}{150} & 61.27 & +-4.5 \\
\hline & & & Pupila - Rima Oris (DERECHO) & & 69.14 & +-4.9 \\
\hline & & & Menton - Punta de la nariz & & 70.39 & +-6.2 \\
\hline & & & Subnasal - Menton & & 58.07 & +-6.2 \\
\hline & & & $\begin{array}{l}\text { Canto externo del ojo - Comisura } \\
\text { Labial (DERECHO) Willis }\end{array}$ & & 70.22 & +-4.1 \\
\hline & & & Pupila - Stomion & & 67.42 & +-4.8 \\
\hline & & & Intercomisural & & 67.78 & +-5.9 \\
\hline & & & Distancia ojo oreja (DERECHO) & & 73.45 & +-4.5 \\
\hline \multirow{6}{*}{ Helal M. 2016} & \multirow{6}{*}{6} & \multirow{6}{*}{19 a 21 años } & Base del Menton - Subnasal & \multirow{6}{*}{120} & 64.6 & +-0.81 \\
\hline & & & Base del mentón - Punta nariz & & 81.0 & +-0.75 \\
\hline & & & $\begin{array}{c}\text { Canto Externo del ojo - comisura } \\
\text { labial (WILIS) }\end{array}$ & & 66.6 & +-0.55 \\
\hline & & & Glabela - Subnasal & & 65.3 & +-0.45 \\
\hline & & & Dedo Índice (derecho) & & 74.8 & +-0.67 \\
\hline & & & $\begin{array}{c}\text { Extremo del pulgar - Extremo del } \\
\text { Índice (Derecho) }\end{array}$ & & 60.6 & +-0.62 \\
\hline & & & Pupila - Rima Oris & & 65.52 & +-5.28 \\
\hline & 1 & & Distancia Ojo - Oreja & 500 & 70.60 & +-4.51 \\
\hline Babu et al $\angle 015$ & 4 & 19 a 56 anos & Largo del pulgar & 500 & 59.32 & +-4.58 \\
\hline & & & Subnasal - Menton & & 66.26 & +-5.04 \\
\hline \multirow{6}{*}{$\begin{array}{l}\text { Majeed M et al } \\
2015\end{array}$} & \multirow{6}{*}{6} & \multirow{6}{*}{18 a 25 años } & Subnasal - Menton & \multirow{6}{*}{300} & 62.70 & \\
\hline & & & Distancia Ojo - Oreja (derecho) & & 69.47 & \\
\hline & & & Intercomisural & & 63.71 & \\
\hline & & & $\begin{array}{c}\text { Canto externo del ojo - ángulo de la } \\
\text { boca }\end{array}$ & & 67.35 & \\
\hline & & & Pupila - Rima Oris & & 65.70 & \\
\hline & & & Interpupilar & & 61.41 & \\
\hline \multirow{3}{*}{$\begin{array}{l}\text { Nasser et al } \\
2016\end{array}$} & \multirow{3}{*}{3} & \multirow{3}{*}{19 a 28 años } & Nariz - Menton punta a punta & \multirow{3}{*}{114} & 69.60 & +-5.32 \\
\hline & & & Subnasal - Menton & & 67.24 & +-4.59 \\
\hline & & & Ojo- Rima Oris & & 70.79 & +-4.01 \\
\hline
\end{tabular}

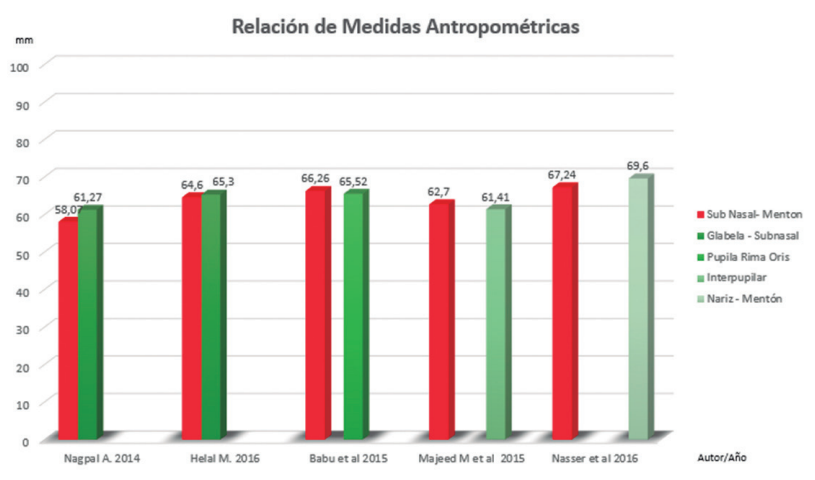

Figura 1. Esquema de medidas antropométricas entre los diferentes estudios realizados. técnicas empleadas para la valoración de la DVO se describen en la Tabla 3 en la cual se determinan sus principales ventajas y desventajas ${ }^{(1)}$.

Las medidas antropométricas ${ }^{(6,12,13,14,15,16,17)}$, o dimensiones faciales ${ }^{(20,21)}$, corresponden a valores que de acuerdo a varios estudios guardan relación entre segmentos del tercio medio y tercio inferior del rostro(6,12,13,14,15) y que pueden servir de referencia o ayuda al clínico para valorar la DVO perdida, la descripción de dichas medidas se muestran en la tabla 4, la determinación gráfica de las mismas se esquematiza en la Figura 2 y los valores representativos de ciertos estudios que comparan dichas medidas se encuentran en la tabla 2.

Seguridad en el aumento de la Dimensión Vertical Oclusal (DVO)

La DVO, determina la proporción facial del tercio inferior con respecto al rostro durante la máxima intercuspidación y está íntimamente relacionada con la $D V P^{(2)}$, es necesaria una evaluación exhaustiva tanto extra como intra oral para evaluar la idoneidad de aumentar la DVO del paciente ${ }^{(1)}$, esta debería ser aumentada en condiciones específicas evaluando la estructura dental remanente, el espacio disponible para la rehabilitación, la oclusión y la necesidad estética ${ }^{(1,4,8)}$, y siempre considerando dos principios básicos: primero: dicho incremento debe partir de un punto de reconstrucción basado en una relación céntrica y segundo: 
Tabla 3. Descripción de técnicas clínicas para valorar a DVO perdida

\begin{tabular}{|c|c|c|c|}
\hline TÉCNICA & DESCRIPCIÓN & VENTAJAS & DESVENTAJAS \\
\hline $\begin{array}{l}\text { Registros Pre } \\
\text { Tratamiento }\end{array}$ & $\begin{array}{l}\text { - Valoración visual de modelos de } \\
\text { diagnóstico antiguos } \\
\text { - Fotografías previas }\end{array}$ & $\begin{array}{l}\text { - Da un valor aproximado de la pérdida } \\
\text { de la altura clínica de las coronas } \\
\text { dentales } \\
\text { - Permite establecer un registro base }\end{array}$ & $\begin{array}{l}\text { Disposición de modelos antiguas al } \\
\text { iniciar el tratamiento }\end{array}$ \\
\hline Evaluación Fonética & $\begin{array}{l}\text {-S, permite medir el espacio } \\
\text { fisiológico del habla } \\
\text { • F, localiza los bordes incisales } \\
\text { delos dientes superiores } \\
\text { - M, localiza la mandíbula en la } \\
\text { posición de reposo }\end{array}$ & $\begin{array}{l}\text { - Reproducible } \\
\text { - Clínicamente Aplicable } \\
\text { - Indica la adaptación del paciente } \\
\text { después de la pérdida de tejido dental } \\
\text { - Indica la relación incisal de los dientes } \\
\text { anteriores } \\
\text { - Localiza el borde incisal de los dientes } \\
\text { antero superiores con respecto al labio } \\
\text { inferior }\end{array}$ & $\begin{array}{l}\text { - Resultados variables en oclusiones } \\
\text { clase II o III } \\
\text { - Representación deficiente de la } \\
\text { pérdida real de la DVO } \\
\text { - De mayor uso en la construcción de } \\
\text { dentaduras completas }\end{array}$ \\
\hline $\begin{array}{l}\text { Relajación del } \\
\text { Paciente }\end{array}$ & $\begin{array}{l}\text { - Posicionamiento Mandibular en } \\
\text { Reposo } \\
\text { - Determina la Dimensión Vertical } \\
\text { Postural (DVP) }\end{array}$ & $\begin{array}{l}\text { - Clínicamente Aplicable } \\
\text { - Visualiza la apariencia facial en reposo } \\
\text { - Asegura el contacto labial }\end{array}$ & $\begin{array}{l}\text { - Alteraciones en la tensión muscular } \\
\text { desencadenan medidas inexactas. }\end{array}$ \\
\hline $\begin{array}{l}\text { Valoración de la } \\
\text { apariencia facial }\end{array}$ & $\begin{array}{l}\text { - Evaluación de los tejidos faciales y } \\
\text { musculatura en reposo }\end{array}$ & $\begin{array}{l}\text { - Clínicamente Aplicable } \\
\text { - Visualiza la apariencia facial en reposo } \\
\text { - Asegura el contacto labial }\end{array}$ & $\begin{array}{l}\text { - Evaluación arbitraria de la estética } \\
\text { facial }\end{array}$ \\
\hline $\begin{array}{l}\text { Evaluación } \\
\text { Radiográfica }\end{array}$ & $\begin{array}{l}\text { - Valoración cefalométrica de la } \\
\text { relación máxilo mandibular }\end{array}$ & $\begin{array}{l}\text { - Muy precisa y Reproducible } \\
\text { • Indica la relación Incisal de los dientes }\end{array}$ & $\begin{array}{l}\text { - Necesidad de equipo adicional } \\
\text { - Utilización de Radiación }\end{array}$ \\
\hline $\begin{array}{l}\text { Evaluación } \\
\text { Neuromuscular }\end{array}$ & $\begin{array}{l}\text { - Registro de la actividad muscular } \\
\text { mediante EMG donde la menor } \\
\text { actividad muscular indica la posición } \\
\text { de reposo mandibular }\end{array}$ & $\begin{array}{l}\text { - Herramienta de uso clínico y de } \\
\text { investigación para la evaluación de la } \\
\text { DVO } \\
\text { - Precisa y Reproducible }\end{array}$ & $\begin{array}{l}\text { - Los dispositivos rara vez están } \\
\text { disponibles en el entorno clínico } \\
\text { - Es necesaria una gran experticia en } \\
\text { el manejo de los instrumentos } \\
\text { - Son necesarias condiciones } \\
\text { controladas y rigurosas para su } \\
\text { registro }\end{array}$ \\
\hline Fuerza Oclusal ${ }^{20}$ & $\begin{array}{l}\text { - Determina la DVP I determinarse la } \\
\text { máxima fuerza de cierre oclusal }\end{array}$ & $\begin{array}{l}\text { - Limitada por la sensación de dolor } \\
\text { percibida por el paciente }\end{array}$ & $\begin{array}{l}\text { - Necesita aditamentos adicionales } \\
\text { como el Bimeter de Boss } \\
\text { - El registro obtenido puede alcanzar } \\
\text { los } 9 \text { mm lo cual lo hace restringido } \\
\text { en su uso }\end{array}$ \\
\hline Test de Deglución ${ }^{21}$ & $\begin{array}{l}\text { - Determinación de la DVO al } \\
\text { momento de deglutir }\end{array}$ & $\begin{array}{l}\text { - Es un patrón idéntico tanto en el } \\
\text { infante edéntulo como en el adulto } \\
\text { edéntulo }\end{array}$ & $\begin{array}{l}\text { - Técnica de difícil interpretación } \\
\text { sobre todo en pacientes edéntulos } \\
\text { - Pueden existir discrepancias de } \\
\text { hasta } 5 \mathrm{~mm} \text { en grupos edentulos }{ }^{20}\end{array}$ \\
\hline $\begin{array}{l}\text { Espacio de } \\
\text { Inoclusión } \\
\text { Fisiológica }^{21}\end{array}$ & $\begin{array}{l}\text { - Corresponde a la diferencia ente la } \\
\text { DVO y DVP }\end{array}$ & $\begin{array}{l}\text { - Medida promedio entre } 1 \text { a } 3 \mathrm{~mm} \\
\text { - Espacio necesario para cumplir con las } \\
\text { funciones fisiológicas }\end{array}$ & $\begin{array}{l}\text { - Estudios demuestran que es una } \\
\text { medida variable dependiendo del tipo } \\
\text { de oclusión del paciente }\end{array}$ \\
\hline
\end{tabular}

la reconstrucción debe estar dentro de los rangos de adaptación neuromuscular del paciente ${ }^{(4)}$, por lo tanto, la decisión del cambio no debe ser tomado a la ligera puesto que involucra la restauración completa de un arco y en algunos casos de los dos ${ }^{(2)}$, este incremento debe ser paulatino, considerándose seguro un aumento de hasta $5 \mathrm{~mm}^{(1,5)}$, el cual es un valor sensible de ser detectado cefalométricamente utilizando el ángulo de la Altura Facial Inferior de Rickets ${ }^{(22)}$, luego del incremento se pueden presentar con frecuencia varios signos y síntomas que causan disconfort en los pacientes ${ }^{(5)}$, y algunos autores incluso lo asocian con problemas temporomandibulares ${ }^{(1,5)}$, estos conceptos están siendo analizados y redefinidos puesto que en una revisión de la literatura Moreno I. y Okenson J. (2015) concluyen que no hay evidencia científica fundamentada en estudios de casos aleatorios o controles longitudinales en el tiempo, que certifiquen claramente la relación del aumento o disminución de la DVO con Trastornos Témporo Mandibulares y que el sistema estomatognático es capaz de adaptarse rápidamente a los moderados cambios oclusales originados por las variaciones de la $\mathrm{DVO}^{(8)}$. Los incrementos de la DVO son seguros y predecibles, y los procedimientos con intervención de aparatología fija proporcionan mejores resultados y con mayor nivel de adaptación al compararlos con los tratamientos de tipo removible ${ }^{(1,5)}$.

\section{CONCLUSIONES Y RECOMENDACIONES}

Dentro de las limitaciones de esta revisión se puede concluir que en la literatura la determinación de la DVO constituye aún un conflicto para el clínico, si bien, es uno de los pasos fundamentales en la evaluación y planificación de la rehabilitación, no hay un método que por sí solo determine con exactitud la medida de la DVO ideal, esto debido a que los estu- 
Esquema Medidas Antropométricas

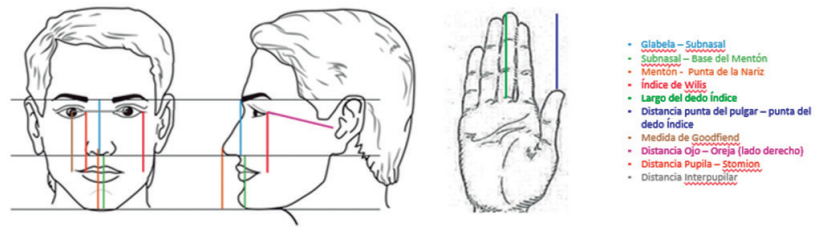

Figura 2. Esquema referencial de la ubicación de las medidas antropométricas del rostro que fueron evaluadas en los diferentes estudios

dios se realizan en diferentes etnias, con diferentes puntos de referencia en los pacientes, no existen métodos para poder comparar eficazmente dichos estudios o a población utilizada no es significativa.

Los incrementos de la DVO de $5 \mathrm{~mm}$ son procedimientos predecibles y seguros que si bien pueden manifestar incomodidades iniciales a los pacientes, estas son auto limitantes y remiten al cabo de pocos días, no hay evidencia científica que respalde la asociación de Trastornos Témporo Mandibulares con el aumento o disminución de la DVO.

Al decidir el aumento de la DVO, se debe partir de un punto estable determinado en una relación céntrica óptima del individuo y preferentemente utilizando aparatología fija ya sea en todo el arco dental completo o si el caso lo requiere de los dos.

Es importante que los valores cefalométricos de los estudios clásicos se verifiquen en la población chilena, en busca de una referencia objetiva que permita establecer las más óptimas relaciones entre los diferentes segmento del rostro, buscando las concordancias de la simetría facial, con ello se podría sugerir la utilización de una medida antropométrica que tenga un alto nivel de sensibilidad al momento de valorar a DVO en esta población.

Entre los métodos descritos y técnicas utilizadas existen muchas variables que deben ser tomadas en cuenta antes de ser aplicadas a cada paciente, como la edad, la etnia, el género o el biotipo facial, es necesario establecer parámetros que determinen cual es la proporción estándar en la población chilena ya sea a base de mediciones antropométricas o cefalométricas que indiquen la armonía de sus elementos y así contribuyan como parámetros guía para la valoración de la DVO.

\section{CONFLICTO DE INTERÉS}

Los autores no tenemos conflictos de interés.
Tabla 4. Medidas antropométricas más utilizadas para determinar la DVO

\begin{tabular}{|ll|}
\hline \multicolumn{1}{|c|}{ MÉTODO } & \multicolumn{1}{c|}{ DESCRIPCIÓN } \\
\hline${\text { Glabela }- \text { Subnasal }^{12}} \begin{array}{l}\text { Medida establecida desde la unión del } \\
\text { borde inferior del septum nasal y el labio } \\
\text { superior(SN) y Glabela que es el punto más } \\
\text { prominente de la línea media ubicado entre } \\
\text { las cejas (G) }\end{array}$ \\
\hline
\end{tabular}

Subnasal - Base del

Establecida desde la unión del borde inferior Mentón ${ }^{12}$ del septum nasal y el labio superior (SN) y la superficie inferior de la barbilla ( Me)

Mentón - Punta de la Distancia entre la superficie inferior de la $\begin{array}{ll}\text { Nariz }^{12} & \text { barbilla ( Me) y el punto más pronun } \\ & \text { ápice de la nariz (punta de la nariz) }\end{array}$

\begin{tabular}{|ll}
\hline Índice de Wilis & Distancia entre el canto externo del ojo y la \\
comisura de la boca
\end{tabular}

\begin{tabular}{|ll}
\hline $\begin{array}{l}\text { Largo del dedo } \\
\text { Índice }{ }^{12}\end{array}$ & $\begin{array}{l}\text { Medida palmar tomada desde la punta del } \\
\text { dedo hasta el pliegue de la mano del mismo } \\
\text { dedo }\end{array}$ \\
\hline
\end{tabular}

Distancia punta del pulgar - punta del dedo Índice ${ }^{12}$

Distancia medida entre el dedo pulgar e índice cundo estos están juntos

$\begin{array}{ll}\begin{array}{l}\text { Medida de } \\ \text { Goodfiend }\end{array} & \begin{array}{l}\text { Distancia entre la pupila del ojo y Rima Oris } \\ \text { (punto de unión en la esquina de los labios) }\end{array}\end{array}$

Distancia Ojo - Oreja
(lado derecho) $^{21}$

Distancia desde la pared anterior del conducto auditivo externo piel a la esquina lateral de la órbita piel (distancia ojo-oreja)

\begin{tabular}{ll}
$\begin{array}{l}\text { Distancia Pupila - } \\
\text { Stomion }\end{array}$ & $\begin{array}{l}\text { Medida establecida desde la pupila hasta } \\
\text { la unión de los labios en la línea media } \\
\text { (Stomion) }\end{array}$ \\
\hline
\end{tabular}

Distancia

Interpupilar ${ }^{16}$
Medida comprendida ente las pupilas de los ojos en posición orto estática del paciente

\section{Bibliografía}

1. Abduo J, Lyons K. Clinical considerations for increasing occlusal vertical dimension: a review. Aust Dent J. 2012;57:2-10.

2. Bachhav V, Aras M. Altering occlusal vertical dimension in functional and esthetic rehabilitation of severely worn dentition. Journal of Oral Health Research. 2010;1(1):2-8.

3. Discacciati JAC, de Souza EL, Vasconcellos WA, Costa SC, Barros VM. Increased vertical dimension of occlusion: signs, symptoms, diagnosis, treatment and options. J Contemp Dent Pract. 2013;14(1):123-128.

4. Chander G, Venkat R. An appraisal on increasing the occlusal vertical dimension in full occlusal rehabilitation and its outcome. J Indian Prosthodont Soc. 2011;11(2):77-81. 5 . Abduo J. Safety of increasing vertical dimensión of occlusion: A systematic review. Quintessence Int. 2012;43:369-380.

6. Nagpal A, Parkash H, Bhargava A, Chittaranjan B. Reliability of different facial measurements for determination of vertical dimension of occlusion in edentulous using accepted facial dimensions recorded from dentulous subjects. J Indian Prosthodont Soc. 2014;14(3):233-242.

7. Carrera C, Larrucea C, Galaz C. Detección de incrementos de dimensión vertical oclusal mediante análisis cefalométrico de Ricketts. Rev Clin Periodoncia Implantol Rehabil Oral. 2010;3(2);79-85.

8. Moreno I, Okeson J, Does altering the occlusal vertical dimension produce temporomandibular disorders? A literature review. J Oral Rehabil. 2015; 42(11):875-82. 9. Gaete $P$, Gaete $M$, Cáceres $M$. Evaluación craneométrica, utilizando el craneometro de Knebelman, de la dimensión vertical oclusal (DVO) obtenida mediante un procedimiento clínico clásico. Rev Dental Chile. 2012;103(2):29-35. 10. Legan $H$, Burstone $C$. Soft tissue cephalometric analysis for orthognathic surgery. J Oral Surg. 1980;38(10):744-751.

11. Celebi A, Tan E, Erhan I, Colak T, Ayyildiz E. Comparison of soft tissue cephalometric norms between Turkish and European-American adults. The ScientificWorld Journal. 2013;2013:806203

12. Helal M, Hasan A. Evaluation of lower facial heights as related to different anthropometric measurements in dentate and completely edentulous subjects. Quintessence Int. 2016;47(1):51-60
13. Babu B, Kumar P, Kumar R, Suwal P, Shresha P, Baral D. An anthropometric study to evaluate the correlation between the occlusal vertical dimension and length of the thumb. Clin Cosmet Investig Dent. 2015;7:33-9

14. Maijed M, Afzal M, Kashif M. Determination of occlusal vertical dimension in a section of Pakistani population using craniofacial measurements. Med Forum 2015; 26(1):55-57.

15. Nasser M, Khalifa N, Amram A. Eye-rima oris distance and its relation to the vertical dimension of occlusion measured by two methods: Anthropometric study in a sample of Yemeni dental students. Eur J Dent. 2016;10:29-33.

16. Ladda R, Kasat V, Bhandari A. A new technique to determine vertical dimension of occlusion from anthropometric measurement of interpupillary distance. J Clin Exp Dent. 2014;6(4):e395-9.

17. Igic M, Krunic N, Aleksov N, Kostic M, Igic A, Petrovic M, et al. Determination of vertical dimension of occlusion by using the phonetic vowel "O" and "E". Vojnosanit Pregl. 2015;72(2):123-131.

18. Quiroga R, Riquelme R, Sierra M, Del Pozo J, Quiroga AR. Determinación de la dimensión vertical oclusal en desdentados totales: comparación de métodos convencionales con el craneómetro de Knebelman. Rev Clin Periodoncia Implantol Rehabil Oral. 2012;5(1):20-24.

19. Quamar K, Munir U, Naeem S. Role of cephalometery in evaluation of vertical dimension. Pakistan Oral \& Dental Journal. 2013;33(1).183-186.

20. Turell AJW. Clinical assessment of vertical dimension. J Prosthet Dent. 2006; 96:79-83.

21. Romo F, Joquera C, Irribarra M. Determinación de la dimensión vertical oclusal a través de la distancia clínica ángulo externo del ojo al Surco Tragus Facial. Rev Dental Chile.2009;100(3) 26-33.

22. Carrera C, Larrucea C, Galaz C. Detection of increases of vertical occlusal dimension by Ricketts cephalometric analysis. Rev Clin Periodoncia Implantol Rehabil Oral. 2010;3(2):79-85. 